\title{
Promotion of epithelial-mesenchymal transition by Frizzled2 is involved in the metastasis of endometrial cancer
}

\author{
YIDING BIAN $^{1 *}$, XINWEN CHANG $^{1 *}$, YUN LIAO $^{2}$, JINGYUN WANG $^{3}$, \\ YIRAN $\mathrm{LI}^{4}, \mathrm{KAI} \mathrm{WANG}^{1}$ and XIAOPING WAN ${ }^{3}$ \\ ${ }^{1}$ Clinical and Translational Research Center, Shanghai First Maternity and Infant Hospital, \\ Tongji University School of Medicine, Shanghai 200040; ${ }^{2}$ Department of Obstetrics and Gynecology, \\ Women's Hospital, School of Medicine, Zhejiang University, Hangzhou, Zhejiang 310006; \\ ${ }^{3}$ Department of Gynecology, Shanghai First Maternity and Infant Hospital, Tongji University School of Medicine, \\ Shanghai 201204; ${ }^{4}$ Department of Obstetrics and Gynecology, Shanghai First People's Hospital \\ Affiliated to Shanghai Jiao Tong University, Shanghai 200080, P.R. China
}

Received February 3, 2016; Accepted March 15, 2016

DOI: 10.3892/or.2016.4885

\begin{abstract}
The Wnt signaling pathway is essential for embryonic development, and genetic alteration in this network is closely correlated with tumorigenesis and progression. Previous research has shown that Wnt receptor Frizzled2 (Fzd2) is elevated in many metastatic cancer cell lines and high grade tumors. Yet, little is known about the Fzd2 expression and activity in human endometrial cancer (EC). In this study, we present evidence of a direct role of Fzd2 in human EC. We found that Fzd2 expression was higher in EC than that in adjacent normal tissues, and was correlated with epithelial-mesenchymal transition markers. Next, it was determined that the stable overexpression of Fzd2 in HEC-1B and Ishikawa cells promoted cell migration and induced an EMT phenotype. Conversely, RNA interference-mediated depletion of Fzd 2 inhibited EC cell migration. Additionally, mechanistic investigation revealed that elevated Fzd2 expression activated canonical Wnt signaling and was blocked by canonical Wnt
\end{abstract}

Correspondence to: Dr Kai Wang, Clinical and Translational Research Center, Shanghai First Maternity and Infant Hospital, Tongji University School of Medicine, 536 Changle Road, Jingan, Shanghai 200040, P.R. China

E-mail: kaiwangcnn@yahoo.com

Dr Xiaoping Wan, Department of Gynecology, Shanghai First Maternity and Infant Hospital, Tongji University School of Medicine, 2669 Gaoke West Road, Pudong New Area, Shanghai 201204, P.R. China

E-mail: wanxiaoping@tongji.edu.cn

*Contributed equally

Key words: endometrial cancer, Frizzled2, epithelial-mesenchymal transition, metastasis, Wnt signaling signaling inhibitor XAV939. However, Fzd2 did not influence the proliferation of EC cells. Thus, Fzd2 may be a potential marker for EC metastasis and a target for future therapies for this disease.

\section{Introduction}

Endometrial cancer (EC) is one of the most common cancers of the female reproductive system with an estimated 54,870 newly diagnosed cases and 10,170 deaths in the USA alone in 2015 (1). In China, the incidence of EC has increased markedly with a higher prevalence in younger women due in part to factors such as obesity and lifestyle changes $(2,3)$. EC is usually diagnosed in early stages $(90 \%)$ and is often successfully treated with surgical intervention (4). However, EC may metastasize to the pelvic, para-aortic lymph nodes or to distant sites via different routes, and most deaths from EC are caused by metastases that are resistant to conventional therapies. Therefore, it is significant to elucidate the molecular mechanism underlying EC metastasis so as to gain insight into better diagnostic and prognostic biomarkers, as well as novel therapies.

Tumor metastasis consists of several steps, all of which are required for the spread of tumor cells (5). Notably, during tumor progression, epithelial-mesenchymal transition (EMT) is activated in certain cancer cells and enables them to acquire cellular characteristics associated with high-grade malignancy, including the capacity to complete various steps in the metastatic cascade (6). In addition, several studies have elucidated a link between EMT and stem cell characteristics and drug resistance, reinforcing the opinion that EMT is closely related to tumor progression $(7,8)$. During EMT, epithelial cells undergo extensive alterations in gene expression patterns, resulting in the loss of apico-basal polarity, fracture of intercellular adhesive junctions, and degradation of basement-membrane components (9). In this way, epithelial cells adopt mesenchymal traits by altering their morphology, cellular architecture, adhesion, and migratory capacity (10). 
However, the mechanisms and pathways that initiate EMT are not comprehensively clear.

EMT is a dynamic procedure and triggered by interactions between extracellular components from the microenvironment and secreted factors, such as the wingless-type MMTV integration site family members (Wnts), transforming growth factor- $\beta$ (TGF- $\beta$ ), fibroblast growth factors, and epidermal growth factors (9). These factors participate in multiple signaling pathways and initiate the expression of downstream transcription factors, such as Snail and Twist, as well as cytokines, such as MMP2 and MMP9. Among the involved signaling pathways, the Wnt signaling pathway plays a critical role in inducing EMT (11-13). Wnt family proteins bind to and activate one or more of the 10 seven-transmembrane Fzd family receptors, playing roles in proliferation, migration, and invasion (14). Previous studies have shown that during EMT, Wnt5a/b ligand and/or its cognate receptor Fzd 2 are generally overexpressed in cell lines derived from late-stage mesenchymal-type cancers, such as melanoma and cancers of the breast, lung, colon, liver, and the gastric tract (15-18). However, whether Wnt5a/b-Fzd2 induces EMT in EC and a mechanistic understanding of signaling pathway regulation have been left unanswered by previous investigations.

This study explored the association between the expression of Wnt receptor Fzd2 and EMT markers in EC tissues and investigated the role of Fzd2 in the regulation of EMT in EC cell lines. The findings shed light on the correlation between Fzd 2 and the promotion of an EMT phenotype and cell migration in EC, and could potentially guide the development of new therapies for EC metastasis.

\section{Materials and methods}

Patients and tissues. Thirteen cases of fresh EC and para-tumor normal endometrial tissues were obtained from Chinese female patients who underwent surgical treatment during 2014 and 2015 at the Shanghai First Maternity and Infant Hospital (Shanghai, China). No patient had undergone endocrine therapy, radiotherapy, or chemotherapy before surgery. This study was approved by the Human Investigation Ethics Committee of the Shanghai First Maternity and Infant Hospital. The samples were collected after written informed consent was obtained from the patients.

Cell culture. The human EC cell lines HEC-1B and Ishikawa were obtained from the American Type Culture Collection (ATCC; Manassas, VA, USA). The cells were cultured in Dulbecco's modified Eagle's medium (DMEM)/F12 (Gibco, Auckland, New Zealand), supplemented with $10 \%$ fetal bovine serum (FBS) (Gibco Life Technologies, Carlsbad, CA, USA) and $100 \mathrm{U} / \mathrm{ml}$ penicillin/streptomycin, and maintained in a $5 \% \mathrm{CO}_{2}$ humidified incubator at $37^{\circ} \mathrm{C}$.

Transient and stable transfection. For stable overexpression of human Fzd2 in EC cells, Fzd2 coding sequences were cloned into lentiviral vectors with Ubi-MCS-3FLAG-SV40-puromycin using Gateway technology (Invitrogen Life Technologies, Carlsbad CA, USA) by GeneChem Biotech Co., Ltd. (Shanghai, China). HEC-1B and Ishikawa cells were infected with nontarget or Fzd2-specific lentiviral particles in 6-well plates in
Table I. Primer sequences for real-time PCR analysis.

\begin{tabular}{ll}
\hline Gene & \multicolumn{2}{c}{ Primer sequence } \\
\hline GAPDH & F: 5'-AGGGCTGCTTTTAACTCTGGT-3' \\
& R: 5'-CCCCACTTGATTTTGGAGGGA-3' \\
N-cadherin & F: 5'-TGCGGTACAGTGTAACTGGG-3' \\
& R: 5'-GAAACCGGGCTATCTGCTCG-3' \\
Vimentin & F: 5'-GACGCCATCAACACCGAGTT-3' \\
& R: 5'-CTTTGTCGTTGGTTAGCTGGT-3' \\
SPP1 & F: 5'-GAAGTTTCGCAGACCTGACAT-3' \\
& R: 5'-GTATGCACCATTCAACTCCTCG-3' \\
Cytokeratin7 & F: 5'-TCCGCGAGGTCACCATTAAC-3' \\
& R: 5'-GCTCTGTCAACTCCGTCTCAT-3' \\
Cytokeratin19 & F: 5'-ACCAAGTTTGAGACGGAACAG-3' \\
& R: 5'-CCCTCAGCGTACTGATTTCCT-3' \\
\hline
\end{tabular}

F, forward; R, reverse.

the presence of Polybrene $(5 \mathrm{mg} / \mathrm{ml})$. The cells were treated with puromycin $(1 \mu \mathrm{g} / \mathrm{ml})$ to generate stable Fzd2-overexpressing clones. The siRNA targeting Frizzled2 (si-Fzd2) and the negative control (si-Ctl) were purchased from Hanyin Biotech (Shanghai, China). The cells were transfected with the siRNA in Opti-MEM using Lipofectamine 2000 (11668-019; both from Invitrogen Life Technologies) according to the manufacturer's instructions.

$R N A$ extraction and $q R T-P C R$. Total RNA was extracted from the cultured cells using Trizol reagent (Invitrogen Life Technologies) and converted into cDNA with the One Step PrimeScript RT reagent kit (Takara, Dalian, China). The gene expression was detected by real-time polymerase chain reaction (PCR) using SYBR Green Master Mix (Takara) on an ABI Prism 7000 thermal cycler (Applied Biosystems, Foster City, CA, USA). The gene expression was calculated using the $2^{-\Delta \Delta \mathrm{Ct}}$ formula and normalized against glyceraldehyde 3-phosphate dehydrogenase (GAPDH). The oligonucleotide primers used for quantitative reverse transcription (qRT)-PCR are listed in Table I. The data were obtained in triplicate from three independent experiments.

Protein extraction and western blot analysis. Total protein was extracted with lysis buffer (Beyotime Biotech, Jiangsu, China) containing a $1 \%$ dilution of the protease inhibitor phenylmethanesulfonyl fluoride (Beyotime Biotech). The protein concentrations were determined using a bicinchoninic acid protein assay kit (Beyotime Biotech). Equal amounts of protein were loaded onto each lane of a SDS-PAGE gel for protein separation and transferred to polyvinylidene fluoride membranes (Millipore, Billerica, MA, USA). The membranes were blocked with $5 \%$ bovine serum albumin for $2 \mathrm{~h}$ and incubated with antibodies against Fzd2 (1 $\mu \mathrm{g} / \mathrm{ml}$; R\&D Systems, Inc., Minneapolis, MN, USA), N-cadherin $(1: 1,000)$, vimentin $(1: 1,000)$, E-cadherin $(1: 1,000)$ (all from Cell Signaling Technology, Danvers, MA, USA), 
A

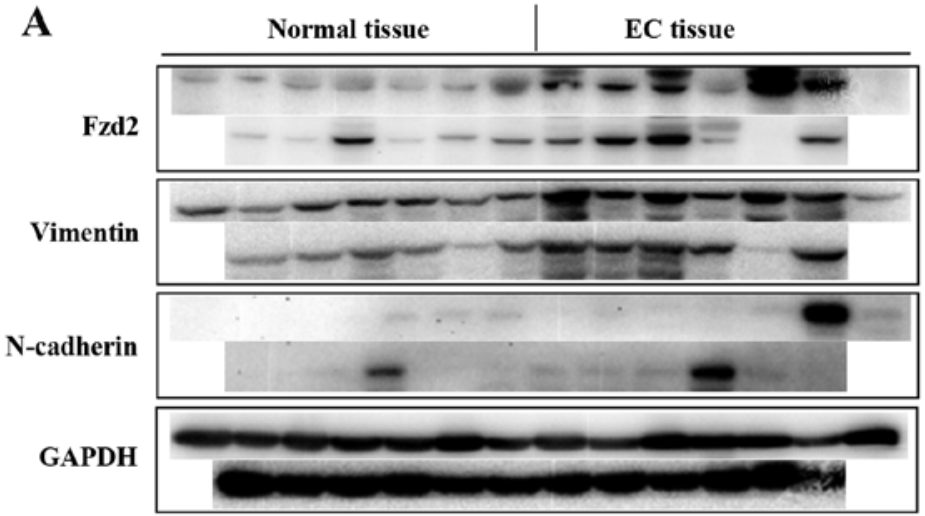

C

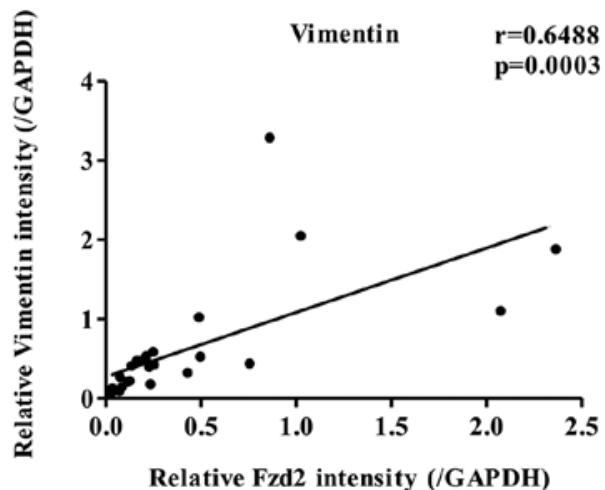

B

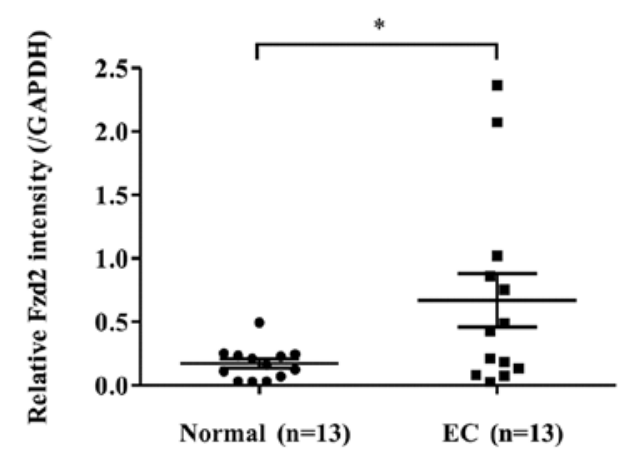

D

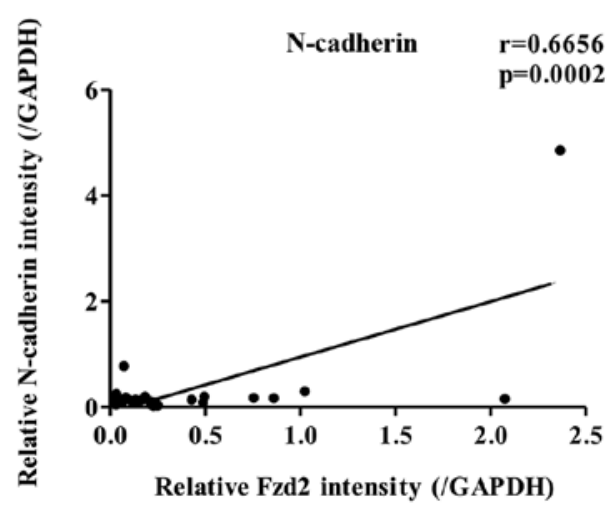

Figure 1. Expression of Fzd2 and EMT markers in EC tissues and paired adjacent normal tissues. (A) Western blot analysis of Fzd2, vimentin, and N-cadherin in EC tissues and paired adjacent normal tissues ( $\mathrm{n}=13$ ). (B) Protein levels of Fzd2 in EC tissues and paired adjacent normal tissues as determined by western blot analysis; GAPDH was included as an internal control ( $\mathrm{P}<0.05)$. Expression correlations between Fzd2 and (C) vimentin and (D) N-cadherin, performed using the Spearman's correlation coefficient test. Fzd2, Frizzled2; EMT, epithelial-mesechymal transition; EC, endometrial cancer.

and GAPDH (1:5,000; Abcam, Cambridge, MA, USA) at $4^{\circ} \mathrm{C}$ overnight. Peroxidase-linked secondary anti-rabbit $(1: 2,000)$ or anti-mouse antibodies $(1: 2,000 ;$ both from Cell signaling Technology) were used to detect the bound primary antibodies, and the blotted proteins were visualized using an enhanced chemiluminescence kit (Pierce Biotechnology, Inc., Rockford, IL, USA). The intensity of protein bands was quantified using ImageJ software (National Institutes of Health, Bethesda, MD, USA). The relative expression of target proteins was described as a ratio relative to the expression of GAPDH, and statistical data from at least three experiments were graphed.

Cell proliferation assay. HEC-1B and Ishikawa cells were seeded into a 96-well plate (3,000 cells/well). Then, $20 \mu \mathrm{l}$ of 3-(4,5-dimethylthiazol-2-yl)-2,5-diphenyltetrazolium bromide (MTT) $(5 \mathrm{mg} / \mathrm{ml}$; Sigma-Aldrich, St. Louis, MO, USA) was added to each well and subsequently incubated at $37^{\circ} \mathrm{C}$ for $1 \mathrm{~h}$. The absorbance was measured at $490 \mathrm{~nm}$ on a plate reader (Bio-Rad, USA). Wells containing known cell numbers $\left(0,1 \times 10^{3}, 2 \times 10^{3}, 5 \times 10^{3}, 10 \times 10^{3}, 20 \times 10^{3}\right.$, or $40 \times 10^{3}$ cells/well; 6 wells/cell density) were treated in a similar fashion to establish standard curves. Each individual experiment was repeated three times in triplicate.

Migration assay. The cells were seeded in 6-well plates and allowed to adhere for $24 \mathrm{~h}$. Confluent monolayer cells were scratched using a $200-\mu 1$ pipette tip and then washed three times with $1 \mathrm{X}$ phosphate-buffered saline to clear cell debris and suspension cells. Fresh serum-free medium was added, and images were captured at 0 and $24 \mathrm{~h}$ at the same position of the wound. For the Transwell assay, a total of $4 \times 10^{4}$ cells were resuspended in $200 \mu \mathrm{l}$ of the serum-free medium and seeded on the top chamber of the Transwell cell culture chambers ( $8 \mu \mathrm{m}$ pore size; Corning Costar, no. 3422). The complete medium $(800 \mu \mathrm{l})$ was added to the bottom chamber as a chemoattractant. After $16 \mathrm{~h}$, the cells that had migrated to the basal side of the membranes were stained with calceinAM $(0.2 \mu \mathrm{g} / \mathrm{ml}$; Invitrogen Life Technologies, no. C3100MP) for $30 \mathrm{~min}$ and counted at a 200 magnification. Recombinant human Wnt5a and Wnt5b (250 ng/ml; R\&D Systems, Inc.) were added to both top and bottom chambers. All experiments were repeated at least three times. The number of cells that had migrated was estimated using MetaMorph image analysis software (Molecular Devices, LLC, Sunnyvale, CA, USA), and the data are expressed as mean average \pm standard deviation $(\mathrm{SD})(\mathrm{n}=3)$.

Construction of reporter plasmids and luciferase assays. T-cell factor/lymphoid enhancer factor (TCF/LEF) reporter M50 Super 8x TOPFlash and M51 Super 8x FOPFlash (TOP Flash mutant) (plasmids \#12456 and \#12457 respectively; Addgene Cambridge, MA, USA) driving the expression of green fluorescent protein (GFP) (TOP/FOP-GFP) were 
A
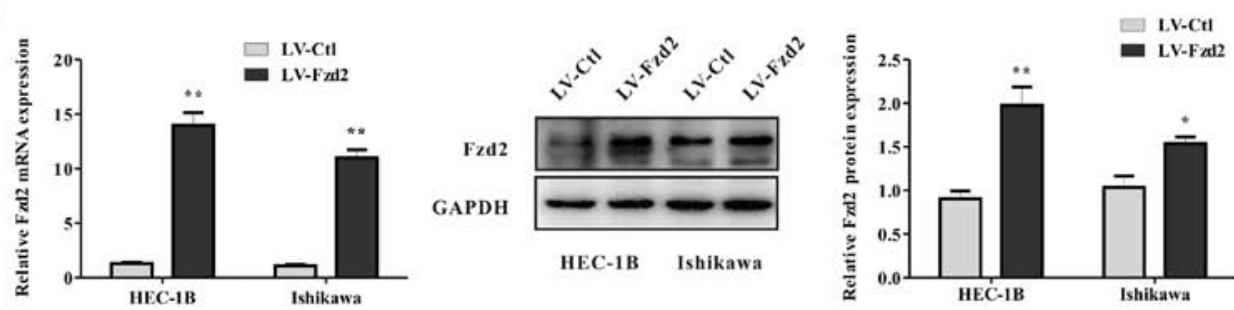

B
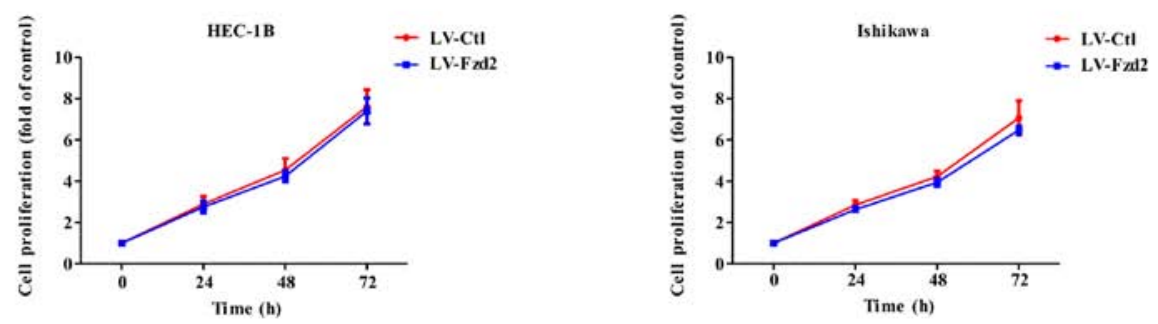

C

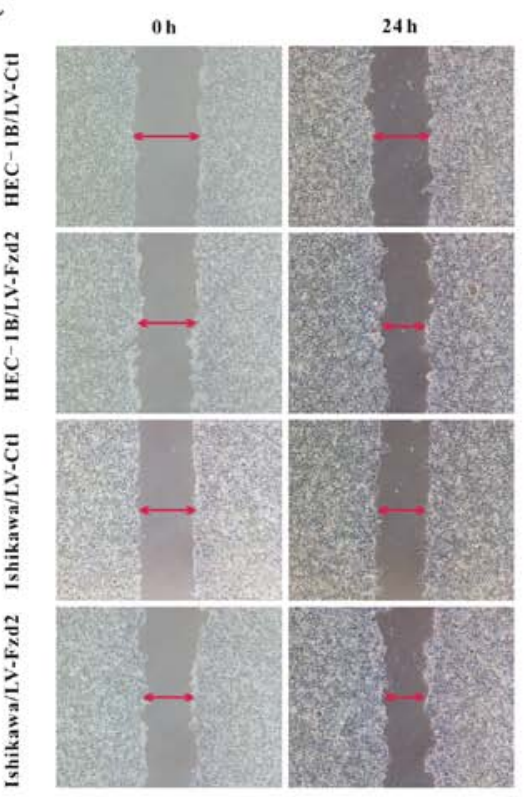

D
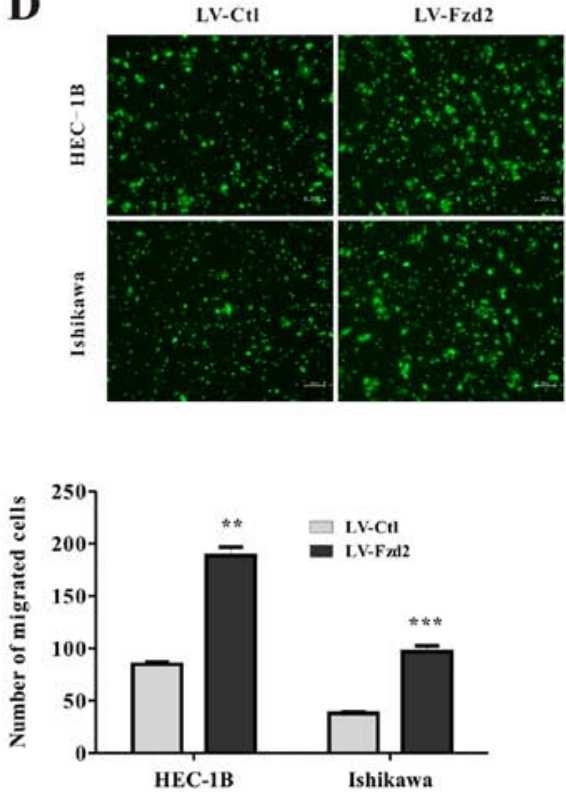

$\mathbf{E}$

Control

+ Wnt5a

$+W n+5 b$
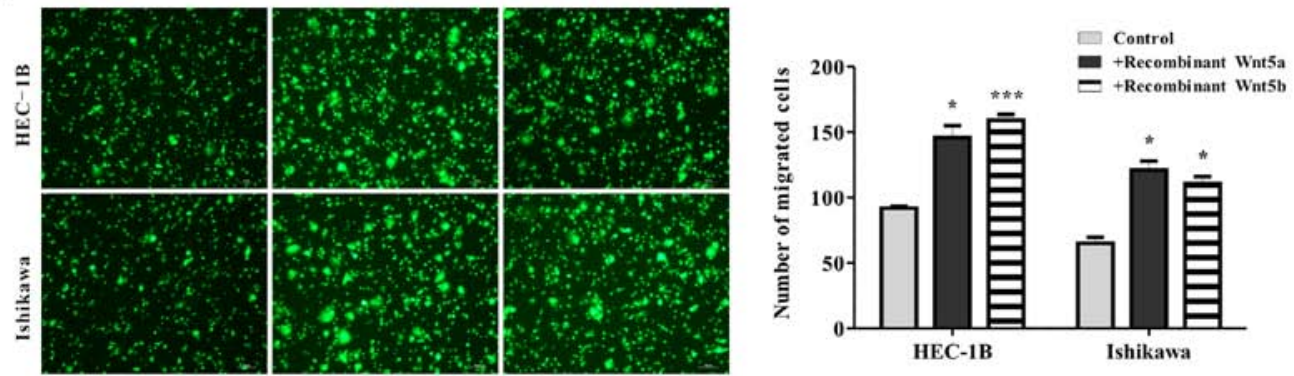

Figure 2. Wnt5-Fzd2 promote the migration of EC cells. (A) Overexpression of Fzd2 in HEC-1B and Ishikawa cells as revealed by qPCR (left) and western blot analysis (middle). Blots were further quantified by densitometry of triplicate blots (right); GAPDH was used as an internal control. (B) An MTT assay was conducted at each time point to quantify the cell viability of the HEC-1B and Ishikawa cells transfected with the control or Fzd2 expression lentiviral vectors. (C) Wound-healing assays of the HEC-1B and Ishikawa cells; representative images were obtained at $\mathrm{x} 40$ magnification. (D) Transwell migration assays of the HEC-1B and Ishikawa cells; representative images were obtained at x200 magnification. The graph shows the number of migrated cells for each treatment group (averaged across five random images). (E) Recombinant human Wnt5a and Wnt5b stimulation increased EC cell migration. Data are expressed as mean \pm SD from one representative experiment of three independent experiments, each performed in triplicate. ${ }^{*} \mathrm{P}<0.05,{ }^{* * *} \mathrm{P}<0.01,{ }^{* * * *} \mathrm{P}<0.001$. Fzd2, Frizzled2; EC, endometrial cancer; EMT, epithelial-mesechymal transition.

gifts from Randall Moon (Cambridge, MA, USA). HEC-1B and Ishikawa cells $\left(2 \times 10^{4}\right)$ were plated in 24-well plates $24 \mathrm{~h}$ before transfection. The cells were co-transfected with $500 \mathrm{ng}$
FOP/TOP reporter plasmid and Renilla luciferase plasmid. The luciferase activity was assayed $24 \mathrm{~h}$ after transfection and measured using Dual-Glo Luciferase reagents (E1531; 
A

B
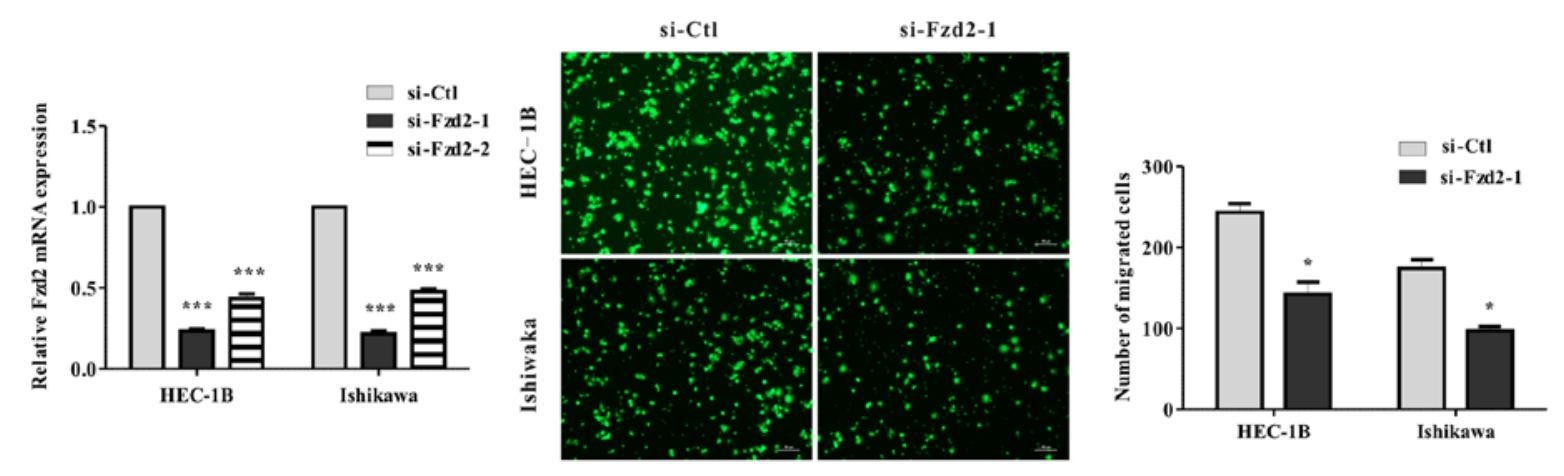

Figure 3. Fzd2 depletion reduces the migration of EC cells. (A) Fzd2 gene silencing efficiency of EC cells by si-Fzd2-1 and si-Fzd2-2. (B) Fzd2 depletion by si-Fzd2-1 reduced the migration of EC cells. Data are expressed as the mean average \pm SD from three independent experiments. ${ }^{*} \mathrm{P}<0.05,{ }^{*} * *<<0.001$. Fzd2, Frizzled2; EC, endometrial cancer.

Promega Corp., Madison, WI, USA). The results were normalized against Renilla activity. All experiments were performed in triplicate.

Statistical analysis. Statistical analyses were performed using the Statistical Package for the Social Sciences software version 17.0 (SPSS, Inc., Chicago, IL, USA). All data are represented as the mean \pm SD. Measurement data were analyzed using unpaired Student's t test or one-way analysis of variance for multiple comparisons. $\mathrm{P}<0.05$ was considered to indicate a statistically significant result.

\section{Results}

Fzd2 expression in EC tissues. A previous study showed Fzd2 overexpression to be associated with many types of human cancers; the present study assessed whether this was also true for EC. Western blot analysis was used to evaluate the expression of Fzd 2 in EC tissues and paired adjacent normal tissues. In a panel of 13 patient tissues, Fzd 2 was overexpressed in EC tissues relative to the level in normal tissues (Fig. 1A and B). Moreover, the expression of Fzd 2 was positively correlated with markers of mesenchymal cells, such as vimentin (VIM) and N-cadherin (CDH2) (Fig. 1A, C and D).

Wnt5-Fzd2 regulates cell migration. To determine whether Fzd2 is required for cell growth or migration, EC cell lines HEC-1B and Ishikawa were stably transfected with lentiviral vectors encoding human Fzd2 (HEC-1B/LV-Fzd2, Ishikawa/LV-Fzd2) and an empty vector as a control (HEC-1B/LV-Ctl, Ishikawa/LV-Ctl). To examine the efficiency of Fzd2 overexpression, the levels of mRNA and protein expression were detected before cellular assays (Fig. 2A). No differences were observed in cell viability between the control and Fzd2-overexpressing cells (Fig. 2B). However, wound-healing and Transwell migration assays both demonstrated that the migration ability of the HEC-1B and Ishikawa cells was markedly increased by 2 - to 3 -fold after Fzd2 overexpression (Fig. 2C and D). Additionally, exposure of HEC-1B and Ishikawa cells to human recombinant protein Wnt5a or Wnt5b also appropriately increased cell migration potential (Fig. 2E). Furthermore, when Fzd2 was depleted in HEC-1B and Ishikawa cells using siRNA (Fig. 3A), a significant reduction in migration (Fig. 3B) was found. Overall, these data showed that Fzd 2 plays a causal role in EC cell motility.

Fzd2 overexpression promotes the EMT phenotype in EC cells. Because Fzd2 levels are correlated with mesenchymal markers in EC tissues, and the processes involved in EMT are closely correlated with cell motility and cancer metastasis, it was hypothesized that Fzd 2 overexpression may drive EMT. To test this, the cellular morphology of the Fzd2-overexpressing EC cells was microscopically examined. HEC-1B/LV-Fzd2 and Ishikawa/LV-Fzd2 cells gained a spindle-shaped morphology and lost cell-cell contacts compared with their control cells, suggesting an EMT phenotype (Fig. 4A). To identify whether this transformation represented EMT, the levels of EMT-associated genes were detected by qRT-PCR and western blot analysis (Fig. 4B and C). Relative to the controls, the levels of epithelial marker E-cadherin in the HEC-1B/LV-Fzd2 and Ishikawa/LV-Fzd2 cells were decreased, whereas the levels of mesenchymal markers CDH2 and VIM were increased. Thus, it was concluded that Fzd2 was involved in the EMT of EC cells.

Fzd2-mediated cell migration is dependent on the canonical Wnt pathway. The activation of the Wnt pathway plays a vital role in EMT during cancer progression. Previous data showed that Fzd 2 could activate $\beta$-catenin-dependent (canonical) signaling by activating the transcription factor TCF, whose activity can be monitored using well-characterized TOP/FOP-GFP reporter plasmids. Overexpression of Fzd 2 in the HEC-1B and Ishikawa cells induced a 2-fold increase in luciferase activity compared with that noted in the vector-only cells (Fig. 5A). Consistently, the levels of $\beta$-catenin protein expression in these cells were also elevated (Fig. 5B). To investigate whether Fzd2-mediated migration depended on the $\beta$-catenin-TCF pathway, HEC-1B/LV-Fzd2 and Ishikawa/LV-Fzd2 cells were treated with an inhibitor of $\beta$-catenin stabilization (XAV939). XAV939 abolished cell migration compared with dimethyl sulfoxide (DMSO) (Fig. 5C). All in all, all these data suggested that Fzd2-mediated cell migration depended on the $\beta$-catenin-TCF pathway in EC cell lines HEC-1B and Ishikawa. 
A
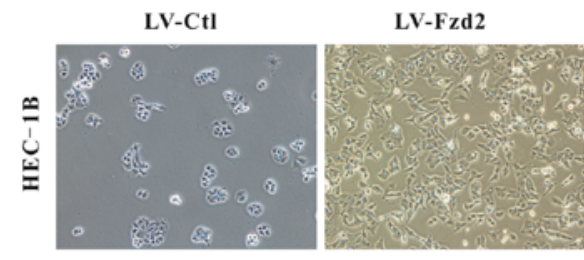

B

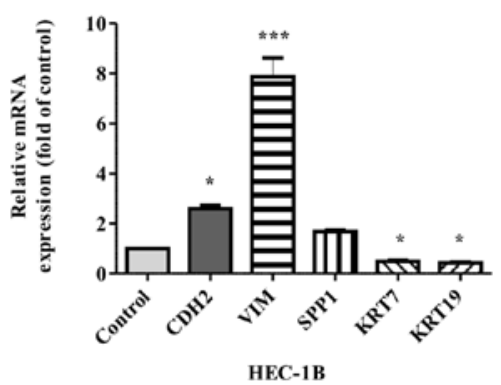

C

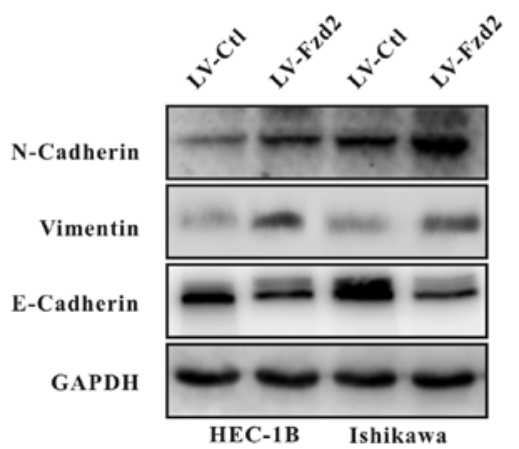

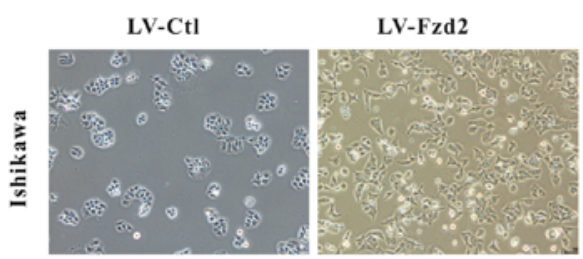

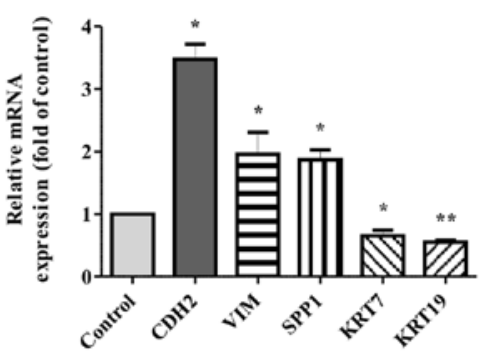

Ishikawa

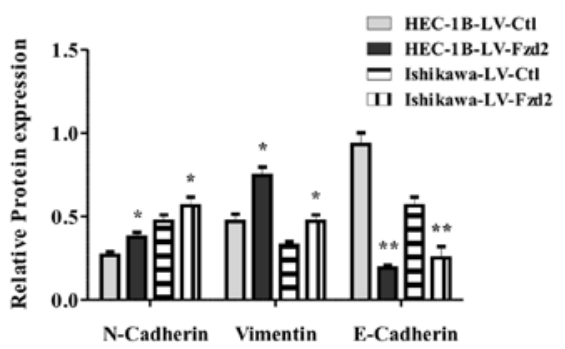

Figure 4. Fzd2 overexpression induces the EMT phenotype in EC cells. (A) Fzd2 overexpression induced a mesenchymal morphology in the HEC-1B and Ishikawa cells (magnification, x200). (B) mRNA levels of EMT markers as analyzed by qRT-PCR in the HEC-1B and Ishikawa cells. GAPDH was included as an internal control. (C) Western blot analysis of EMT-related markers in the HEC-1B and Ishikawa cells (left), and blots were further quantified by densitometry of triplicate blots (right). GAPDH was used as an internal control. ${ }^{*} \mathrm{P}<0.05,{ }^{* * *} \mathrm{P}<0.01,{ }^{* * * *} \mathrm{P}<0.001$.
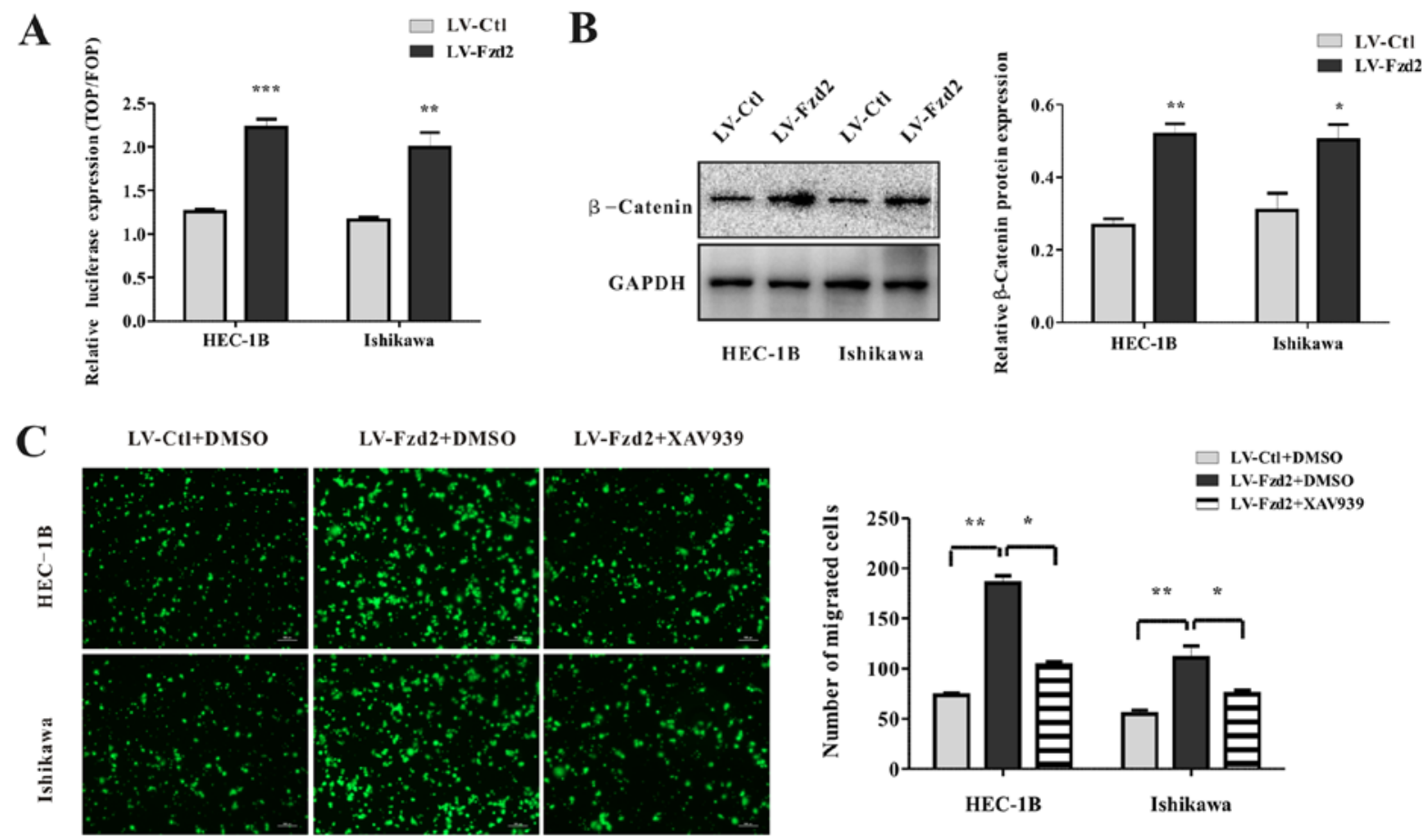

Figure 5. Fzd2-mediated cell migration is dependent on the canonical Wnt pathway. (A) Overexpression of Fzd2 in the HEC-1B and Ishikawa cells induced an increase in luciferase activity compared with the vector-only cells. (B) Western blot analysis of $\beta$-catenin in the HEC-1B and Ishikawa cells. (C) Canonical pathway inhibitor XAV939 $(10 \mu \mathrm{M})$ abolished cell migration after pretreatment for $72 \mathrm{~h}$. Data are expressed as the mean $\pm \mathrm{SD}$ from one representative experiment of three independent experiments. ${ }^{*} \mathrm{P}<0.05,{ }^{* * *} \mathrm{P}<0.01,{ }^{* * * *} \mathrm{P}<0.001$. Fzd2, Frizzled2. 


\section{Discussion}

Tumor metastasis and dissemination are the leading causes of death in EC and as much as $90 \%$ of cancer-associated mortality in general (19). Regrettably, the progress in developing efficient strategies specifically targeting tumor metastasis or cells with metastatic potential has been limited (20). Enhancing the understanding of the molecular mechanisms of the metastatic process might improve the clinical management and outcomes of patients with the disease.

Malignant epithelial tumor cells can disseminate from the primary tumor and invade distant organs through a variety of mechanisms. EMT is considered to be an important means of tumor metastasis in many common cancers, including EC (21). Previous studies have indicated that the activation of Fzd2-mediated signaling might be important in metastatic and late-stage cancers, and a high expression of Fzd2 and its ligand Wnt5a could be a potential marker of poor outcome of patients with hepatocellular carcinoma and prostate cancer, respectively $(17,18)$ The present results are consistent with previous findings and prove for the first time that Fzd2 plays an important role in tumorigenesis and acquisition of the metastatic phenotype in EC. This study found that overexpression of Fzd2 in EC cell lines HEC-1B and Ishikawa could promote cell migration potential and an EMT phenotype with an increase in E-cadherin and concomitant reduction in CDH2 and VIM. The loss of E-cadherin protein appears to be a crucial step, reducing cell-to-cell adhesion and destabilizes the epithelial architecture (22). Additionally, even with limited patient numbers, Fzd2 was overexpressed and correlated with EMT markers in EC tissues relative to paired normal tumor-adjacent tissues. Although these studies are not sufficient to confirm Fzd2 as a prognostic factor for EC, the expression data combined with functional data indicate that Fzd2 may serve as a target for anticancer therapy.

However, how Fzd2 regulates EMT transition in EC development remains unknown and must be further investigated. The Wnt signaling pathway is highly conserved and regulates the specification of cell fate, stem cell maintenance, and initiation of EMT $(14,23)$. The present results showed that Fzd2 overexpression activated the canonical Wnt signaling pathway and increased the $\beta$-catenin protein level. As a result, stable, nonphosphorylated $\beta$-catenin accumulated and translocated into the nucleus, binding to the $\mathrm{N}$ terminus of TCF/LEF transcription factors (24). The TCF/LEF reporter that indicates the expression of fluorescent protein (TOP-GFP) provides direct evidence for the activation of the canonical Wnt signaling. In the present study, Fzd2 overexpression significantly enhanced the luciferase expression of the TOP/FOP reporter plasmid, indicating the dependence on the canonical Wnt pathway. Exposing EC cells to $\beta$-catenin stabilization antagonist XAV939 did affect Fzd2-mediated cell migration, further suggesting the dependence on the canonical Wnt pathway. However, previous studies have shown that Wnt5-Fzd2 activates both $\beta$-catenin-dependent (canonical) and $\beta$-catenin-independent (noncanonical) signaling (25-27). Moreover, at some stage of tumor progression, a switch appears to arise between $\beta$-catenin-dependent and $\beta$-catenin-independent signaling, consistent with the finding that XAV939 could not fully reverse the Fzd2-mediated cell migration. Overall, further investigations are needed to prove that alternative signaling pathways are involved in Fzd2-mediated cell migration and EMT.

Once the cells pass through EMT, an ongoing autocrine Wnt signaling could be established to maintain their residence in the resulting mesenchymal state. However, the concomitant secreted inhibitors of the Wnt signaling pathway, such as DKK1 and SFRP1, would destabilize the autocrine loop. Previous data showed that EMT could be induced by reducing the levels of secreted inhibitors (28). Thus, it remains to be demonstrated whether such a mechanism operates in EC and is critical to EMT induction. Furthermore, a series of additional factors have been found capable of inducing EMT in various types of epithelial cells, including TGF- $\beta$, Notch, Sonic hedgehog, and multiple growth factors secreted into the microenvironment of tumor cells. All things considered, it is also possible to reveal an alternative mechanism in the tumor microenvironment for inducing and maintaining the mesenchymal state in EC.

A delicate balance between estrogen and progesterone signaling underlies the normal functioning of the female reproductive tract and menstrual cycle. The development of EC, especially Type I, is correlated with estrogen excess (29). Previous studies have shown that estrogen enhances Wnt/ $\beta$-catenin signaling in the proliferative phase during the menstrual cycle, while progesterone inhibits Wnt/ $\beta$-catenin signaling, restraining proliferative actions of estrogens in the secretory phase $(30,31)$. Thus, when exposed to enhanced or unopposed estrogen signaling, the constitutive activation of Wnt/ $\beta$-catenin signaling in endometrium would trigger endometrial hyperplasia, which may develop further into EC.

Although surgery is the standard treatment for early-stage EC patients, patients with lymph node or distant-organ metastases also require chemoradiotherapy and often have poor clinical outcomes. Therefore, identifying biomarkers correlated with cell metastatic potential might help to optimize treatment strategies. This study found that Fzd2 levels in EC tissues are positively correlated with the markers of EMT. Furthermore, our findings concerning EC cells showed that Fzd2 overexpression promoted the EMT phenotype, and these effects involved the activation of the Wnt/ $\beta$-catenin pathway. Thus, Fzd2 might be a potential marker for EC metastasis and a target for future therapies for this disease.

\section{Acknowledgements}

This study was supported by the National Natural Science Foundation of China (no. 81172476, 81272885 and 81472427), the Science and Technology Commission of Shanghai Municipality (no. 13JC1404501), the Doctoral Fund of Ministry of Education of China (no. 20120073110090), the Program for Young Excellent Talents in Tongji University (no. 1400813).

\section{References}

1. Siegel RL, Miller KD and Jemal A: Cancer statistics, 2015. CA Cancer J Clin 65: 5-29, 2015.

2. Xu WH, Xiang YB, Zhang X, Ruan Z, Cai H, Zheng W and Shu XO: Association of dietary glycemic index and glycemic load with endometrial cancer risk among Chinese women. Nutr Cancer 67: 89-97, 2015. 
3. Gao J, Yang G, Wen W, Cai QY, Zheng W, Shu XO and Xiang YB Impact of known risk factors on endometrial cancer burden in Chinese women. Eur J Cancer Prev 25: 329-334.

4. Colombo N, Preti E, Landoni F, Carinelli S, Colombo A, Marini C and Sessa C; ESMO Guidelines Working Group: Endometrial cancer: ESMO Clinical Practice Guidelines for diagnosis, treatment and follow-up. Ann Oncol 24 (Suppl 6): vi33-vi38, 2013.

5. Chambers AF, Groom AC and MacDonald IC: Dissemination and growth of cancer cells in metastatic sites. Nat Rev Cancer 2 : 563-572, 2002

6. Brabletz T, Jung A, Spaderna S, Hlubek F and Kirchner T: Opinion: Migrating cancer stem cells-an integrated concept of malignant tumour progression. Nat Rev Cancer 5: 744-749, 2005

7. Singh A and Settleman J: EMT, cancer stem cells and drug resistance: An emerging axis of evil in the war on cancer. Oncogene 29: 4741-4751, 2010.

8. Mani SA, Guo W, Liao MJ, Eaton EN, Ayyanan A, Zhou AY, Brooks M, Reinhard F, Zhang CC, Shipitsin M, et al: The epithelial-mesenchymal transition generates cells with properties of stem cells. Cell 133: 704-715, 2008 .

9. Yang J and Weinberg RA: Epithelial-mesenchymal transition: At the crossroads of development and tumor metastasis. Dev Cell 14 818-829, 2008

10. Lee JM, Dedhar S, Kalluri R and Thompson EW: The epithelial-mesenchymal transition: New insights in signaling, development, and disease. J Cell Biol 172: 973-981, 2006.

11. Moon RT, Kohn AD, De Ferrari GV and Kaykas A: WNT and $\beta$-catenin signalling: Diseases and therapies. Nat Rev Genet 5 : 691-701, 2004

12. Wu ZQ, Li XY, Hu CY, Ford M, Kleer CG and Weiss SJ: Canonical Wnt signaling regulates Slug activity and links epithelial-mesenchymal transition with epigenetic breast cancer 1, early onset (BRCA1) repression. Proc Natl Acad Sci USA 109: 16654-16659, 2012.

13. Chang YW, Su YJ, Hsiao M, Wei KC, Lin WH, Liang CL, Chen SC and Lee JL: Diverse targets of $\beta$-catenin during the epithelial-mesenchymal transition define cancer stem cells and predict disease relapse. Cancer Res 75: 3398-3410, 2015.

14. Willert K, Brown JD, Danenberg E, Duncan AW, Weissman IL, Reya T, Yates JR III and Nusse R: Wnt proteins are lipid-modified and can act as stem cell growth factors. Nature 423: 448-452, 2003

15. Zhang Y, Du J, Zheng J, Liu J, Xu R, Shen T, Zhu Y, Chang J, Wang $\mathrm{H}$, Zhang Z, et al: EGF-reduced Wnt5a transcription induces epithelial-mesenchymal transition via Arf6-ERK signaling in gastric cancer cells. Oncotarget 6: 7244-7261, 2015.

16. Dissanayake SK, Wade M, Johnson CE, O'Connell MP, Leotlela PD, French AD, Shah KV, Hewitt KJ, Rosenthal DT, Indig FE, et al: The Wnt5A/protein kinase $\mathrm{C}$ pathway mediates motility in melanoma cells via the inhibition of metastasis suppressors and initiation of an epithelial to mesenchymal transition. J Biol Chem 282: 17259-17271, 2007.
17. Yamamoto $\mathrm{H}$, Oue N, Sato A, Hasegawa $\mathrm{Y}$, Yamamoto $\mathrm{H}$, Matsubara A, Yasui W and Kikuchi A: Wnt5a signaling is involved in the aggressiveness of prostate cancer and expression of metalloproteinase. Oncogene 29: 2036-2046, 2010.

18. Gujral TS, Chan M, Peshkin L, Sorger PK, Kirschner MW and MacBeath G: A noncanonical Frizzled2 pathway regulates epithelial-mesenchymal transition and metastasis. Cell 159: 844-856, 2014.

19. Weigelt B, Peterse JL and van 't Veer LJ: Breast cancer metastasis: Markers and models. Nat Rev Cancer 5: 591-602, 2005.

20. Sleeman J and Steeg PS: Cancer metastasis as a therapeutic target. Eur J Cancer 46: 1177-1180, 2010.

21. Thiery JP, Acloque H, Huang RY and Nieto MA: Epithelial-mesenchymal transitions in development and disease. Cell 139: 871-890, 2009.

22. Wheelock MJ and Johnson KR: Cadherins as modulators of cellular phenotype. Annu Rev Cell Dev Biol 19: 207-235, 2003.

23. Klaus A and Birchmeier W: Wnt signalling and its impact on development and cancer. Nat Rev Cancer 8: 387-398, 2008.

24. Clevers $\mathrm{H}$ : Wnt/ $\beta$-catenin signaling in development and disease. Cell 127: 469-480, 2006.

25. Zhang Y, Tu C, Zhang D, Zheng Y, Peng Z, Feng Y, Xiao S and $\mathrm{Li} \mathrm{Z}$ : Wnt $/ \beta$-catenin and $\mathrm{Wnt} 5 \mathrm{a} / \mathrm{Ca}^{2+}$ pathways regulate proliferation and apoptosis of keratinocytes in psoriasis lesions. Cell Physiol Biochem 36: 1890-1902, 2015.

26. Grumolato L, Liu G, Mong P, Mudbhary R, Biswas R, Arroyave R, Vijayakumar S, Economides AN and Aaronson SA: Canonical and noncanonical Wnts use a common mechanism to activate completely unrelated coreceptors. Genes Dev 24: 2517-2530, 2010.

27. Zhang E, Li Z, Xu Z, Duan W, Sun C and Lu L: Frizzled2 mediates the migration and invasion of human oral squamous cell carcinoma cells through the regulation of the signal transducer and activator of transcription-3 signaling pathway. Oncol Rep 34: 3061-3067, 2015.

28. Scheel C, Eaton EN, Li SH, Chaffer CL, Reinhardt F, Kah KJ, Bell G, Guo W, Rubin J, Richardson AL, et al: Paracrine and autocrine signals induce and maintain mesenchymal and stem cell states in the breast. Cell 145: 926-940, 2011.

29. Kandoth C, Schultz N, Cherniack AD, Akbani R, Liu Y, Shen H, Robertson AG, Pashtan I, Shen R, Benz CC, et al; Cancer Genome Atlas Research Network: Integrated genomic characterization of endometrial carcinoma. Nature 497: 67-73, 2013.

30. Wang Y, van der Zee M, Fodde R and Blok LJ: Wnt/B-catenin and sex hormone signaling in endometrial homeostasis and cancer. Oncotarget 1: 674-684, 2010.

31. Wang Y, Hanifi-Moghaddam P, Hanekamp EE, Kloosterboer HJ, Franken P, Veldscholte J, van Doorn HC, Ewing PC, Kim JJ, Grootegoed JA, et al: Progesterone inhibition of Wnt $/ \beta$-catenin signaling in normal endometrium and endometrial cancer. Clin Cancer Res 15: 5784-5793, 2009. 